\title{
Risk of ischemic stroke during the initiation period of $\alpha$-blocker therapy among older men
}

\author{
Chao-Lun Lai MD PhD, Raymond Nien-Chen Kuo PhD, Ho-Min Chen MS, Ming-Fong Chen MD PhD, \\ K. Arnold Chan MD ScD, Mei-Shu Lai MD PhD
}

\begin{abstract}
Background: Alpha-blockers are notorious for their first-dose effect of acute hypotension during the early initiation period. Because acute cerebral hypoperfusion may precipitate an episode of ischemic stroke, we aimed to provide a quantitative estimate of the risk of ischemic stroke during the early initiation period of $\alpha$-blocker therapy, using a selfcontrolled case series design.
\end{abstract}

Methods: We identified all men aged 50 years or more as of 2007 who were incident users of $\alpha$-blockers and had a diagnosis of ischemic stroke during the 2007-2009 study period using claims data from Taiwan's National Health Insurance claims database. The first day on which the $\alpha$-blocker was prescribed was the index date. We partitioned different risk periods according to their relationship to the index date (pre-exposure risk periods 1 and $2=\leq 21 \mathrm{~d}$ and 22-60 d before index date, respectively; post-exposure risk periods 1 and $2=\leq 21 \mathrm{~d}$ and
22-60 d after index date, respectively); the remainder of the study period was defined as the unexposed period. We estimated the incidence rate ratio (IRR) of ischemic stroke in each risk period relative to the unexposed period using a conditional Poisson regression model.

Results: A total of 7502 men were included. Compared with the risk in the unexposed period, the risk of ischemic stroke was increased in post-exposure risk period 1 among all patients in the study population (adjusted IRR $1.40,95 \%$ confidence interval $[\mathrm{Cl}], 1.22-1.61)$ and among patients without concomitant prescriptions for other antihypertensive agents (adjusted IRR 2.11, 95\% Cl 1.73-2.57).

Interpretation: Alpha-blocker therapy was associated with an increased risk of ischemic stroke during the early initiation period, especially among patients who were not taking other antihypertensive agents.
$\mathrm{A}$ cute ischemic stroke is usually classified as thrombosis or embolism. ${ }^{1}$ However, acute cerebral hypoperfusion can also precipitate a specific type of ischemic stroke called hemodynamic stroke. ${ }^{2-4}$

Alpha-blockers are strong vasodilators originally classified as antihypertensive agents. Their effectiveness in relieving symptoms in the lower urinary tract has led to their use for the treatment of benign prostatic hyperplasia since $1976 .{ }^{5}$ The Antihypertensive and Lipid-Lowering Treatment to Prevent Heart Attack Trial (ALLHAT) showed that $\alpha$-blockers were inferior to other classes of drugs as the first-line therapy for hypertension. ${ }^{6}$ Currently, $\alpha$-blockers are most commonly prescribed for the treatment of benign prostatic hyperplasia. ${ }^{5}$ Nevertheless, they have inherent adverse cardiovascular effects, including dizziness and hypotension, especially soon after initiation (known as the first-dose effect), because of their effects on lowering blood pressure. ${ }^{5,7,8}$ Researchers have reported an increase in the risk of hypotension-related adverse events, including hypotension, syncope and fractures, during the initiation period of $\alpha$-blocker therapy..$^{9}$ Our research group found that the use of $\alpha$-blockers was associated with an increased risk of hip and femur fractures during the early initiation period among older men without hypertension. ${ }^{10}$ Whether the acute hypotensive effect of $\alpha$-blockers can precipitate the episode of ischemic stroke has not been clarified in a large-scale study, although a case of acute hemiparesis after one dose of doxazosin has been reported. ${ }^{11}$

The aim of this study was to provide a quantitative estimate of the risk of ischemic stroke during the early initiation period of $\alpha$-blocker therapy among older men. We used a self-controlled case series design based on claims data from Taiwan's National Health Insurance claims database.

\section{Methods}

\section{Data source}

The National Health Insurance Research claims database has been described previously. ${ }^{10,12}$ To
Competing interests: None declared.

This article has been peer reviewed.

Accepted: Oct. 26, 2015

Online: Dec. 7, 2015

Correspondence to:

Chao-Lun Lai,

chaolunlai@ntu.edu.tw; and Mei-Shu Lai, mslai@ntu.edu.tw

CMAJ 2016. DOI:10.1503 /cmaj.15062 
comply with Taiwanese privacy regulations, all personal identifiers are encrypted and data can be analyzed only anonymously. The protocol for this study was approved by the Institutional Review Board of the National Taiwan University Hospital, which waived requirement for informed consent.

\section{Study design and cohort definition}

We used claims data from the National Health Insurance Research claims database for the period 2006-2009 and applied a self-controlled case series design. ${ }^{10,13,14} \mathrm{We}$ identified all men aged 50 years or more as of Jan. 1, 2007, who had continuous coverage under the National Health Insurance program for at least 12 months. The study period for each case was defined as the period from Jan. 1, 2007, to Dec. 31, 2009. We selected men who were incident users of $\alpha$-blockers and had a diagnosis of ischemic stroke within the 3 -year study period. We excluded those who had any diagnosis concerning ischemic stroke or any prescription of $\alpha$-blocker in the baseline period (Jan. 1, 2006, to Dec. 31, 2006). Only the first event of ischemic stroke was included in the analysis. ${ }^{14}$ Because of the different clinical implications between use of antiplatelet or anticoagulant agents before (primary prevention) and after (secondary prevention) the incidence of ischemic stroke, we excluded patients who were prescribed any antiplatelet or anticoagulant agent before the incidence of ischemic stroke. This exclusion also increased the accuracy of identifying incident ischemic strokes with our study design.

\section{Definitions of exposures and events}

We identified the use of $4 \alpha$-blockers: terazosin, doxazosin, tamsulosin and alfuzosin. We defined ischemic stroke according to the presence of International Classification of Diseases, Ninth Revision, Clinical Modification (ICD-9-CM) codes 433.x1, 434.x1, 435.9, 436, 437.1x or $437.9 \mathrm{x}$ in the discharge diagnoses in the inpatient records of the National Health Insurance Research claims database. ${ }^{15}$ The diagnosis of acute ischemic stroke in the database has been validated recently. ${ }^{16}$

\section{Study timeframe}

In the database's outpatient records, each drug dispensed at outpatient clinics or pharmacies is included, with the corresponding date of dispensing. All drugs prescribed during hospital stays, however, are recorded in the database's inpatient records, with only the dates of hospital admission and discharge. Therefore, we defined the index date either as the dispensing date of $\alpha$-blocker therapy identified from outpatient records or as the date of hospital admission if the first dose of $\alpha$-blocker was prescribed during the hospital stay. Because all patients with ischemic stroke were identified from the inpatient records, we presumed that the dates of hospital admission were the dates of occurrence of ischemic stroke.

We operationally defined the early initiation period ( $\leq 21 \mathrm{~d}$ after the index date) as postexposure risk period 1 and the 22-60 days after the index date as post-exposure risk period $2 .{ }^{17}$ To determine whether cases of exposure were event-dependent, we also defined the 21 days before the index date as pre-exposure risk period 1 and the 22-60 days before the index date as preexposure risk period 2. ${ }^{14,18}$ The times before and after the pre- and post-exposure risk periods in the 3-year study period were defined as the unexposed periods (Figure 1). For analysis, we grouped the 2 unexposed periods into 1 unexposed period because they were far away from the index date, they conveyed no acute hypotensive effect of $\alpha$-blockers, and the crude incidence rates of ischemic stroke in the 2 unexposed periods were similar (see Table $\mathrm{S} 1$ in Appendix 1, available at www.cmaj.ca/lookup/ suppl/doi:10.1503/cmaj.150624/-/DC1). Because we could not determine the true temporal relationship between initial exposure and the event for cases occurring on the same day, we excluded the index date during partition of the risk periods and included it in the unexposed period in the primary analysis for all the participants to get more conservative estimates. ${ }^{13,18}$

\section{Baseline characteristics}

We evaluated the baseline comorbidities of the study population using the Elixhauser Comorbidity Index ${ }^{19}$ and coded them as binary vari-

\begin{tabular}{|c|c|c|c|c|c|}
\hline \multicolumn{3}{|c|}{ Pre-exposure period } & \multicolumn{2}{|c|}{ Post-exposure period } & \multirow[b]{2}{*}{ Unexposed } \\
\hline Unexposed & -22 to $-60 d$ & -1 to $-21 d$ & $\leq \mathbf{2 1 d}$ & 22 to $60 \mathrm{~d}$ & \\
\hline Jan. 1, 2007 & $\begin{array}{c}\text { Risk } \\
\text { period } 2\end{array}$ & $\begin{array}{c}\text { Risk } \\
\text { period } 1\end{array}$ & $\begin{array}{c}\text { Risk } \\
\text { period } 1\end{array}$ & $\begin{array}{c}\text { Risk } \\
\text { period } 2\end{array}$ & Dec. 31, 2009 \\
\hline
\end{tabular}

Figure 1: Pictorial representation of the study design. 
ables. We determined the specific comorbidity as the presence of corresponding diagnoses in at least 2 outpatient visits or at least 1 inpatient record within the 12-month period before the index date. Only items with a prevalence of more than $2 \%$ were retained.

\section{Statistical analysis}

Using the conditional Poisson regression model, we compared the incidence rates of ischemic stroke in various risk periods with the rates in the unexposed period and presented the results as incidence rate ratios (IRRs). ${ }^{10,13,14,17,18}$ The key advantage of a self-controlled case series design is that time-invariant confounders, such as sex, location, genetics and underlying state of health, are controlled for implicitly. ${ }^{14}$ In the conditional Poisson regression model, we adjusted only for age at the index date and development of atrial flutter or fibrillation (ICD-9-CM code 427.3), which was included as a time-varying term.

Because the acute hypotension-related adverse events associated with $\alpha$-blockers may be modified by the status of concomitant antihypertensive treatment, ${ }^{9,10}$ we repeated the primary analysis with stratification according to whether patients were taking concomitant antihypertensive therapy on the index date. We also performed a stratification analysis according to different $\alpha$-blockers administered.

We performed 2 sensitivity analyses to test the robustness of our results. In the first analysis, we included the index date in pre-exposure risk period 1 , on the assumption that all patients whose index date and stroke event occurred on the same day had their $\alpha$-blocker therapy prescribed during the hospital stay after a preceding ischemic stroke. In the second analysis, we excluded all patients who began their $\alpha$-blocker therapy during a hospital admission (regardless of whether it was the same admission for stroke).

We repeated the same design as the primary analysis for patients newly prescribed diuretics and calcium-channel blockers, to explore whether the observed results for $\alpha$-blockers represented a possible group effect of antihypertensive agents.

We performed all analyses using SAS software, version 9.2 (SAS Institute Inc.).

\section{Results}

We identified a total of 7502 patients who fulfilled our study criteria (1663 were prescribed terazoin, 2252 doxazosin [1493 standard formula and 759 modified-release formula], 3108 tamsulosin and 479 alfuzosin). The mean age was 71.0 years at the index date of $\alpha$-blocker therapy and at the first occurrence of ischemic stroke (Table S2 in Appendix 1). Most of the ischemic strokes occurred within the 3-month period around the index date (Figure S1 in Appendix 1).

For the whole study population, the incidence of ischemic stroke was significantly higher in post-exposure risk period 1 than in the unexposed period (adjusted IRR 1.40, 95\% confidence interval [CI] 1.22-1.61). It was also significantly higher in both pre-exposure risk periods (preexposure risk period 1: adjusted IRR 2.87, 95\% CI 2.60-3.17; pre-exposure risk period 2: adjusted IRR $1.99,95 \%$ CI 1.82-2.18). Conversely, the incidence in post-exposure risk period 2 was lower than in the unexposed period (adjusted IRR 0.80, 95\% CI 0.70-0.92) (Table 1).

Among patients with concomitant prescriptions for antihypertensive agents, the incidence of ischemic stroke in post-exposure risk period 1 was similar to that in the unexposed period

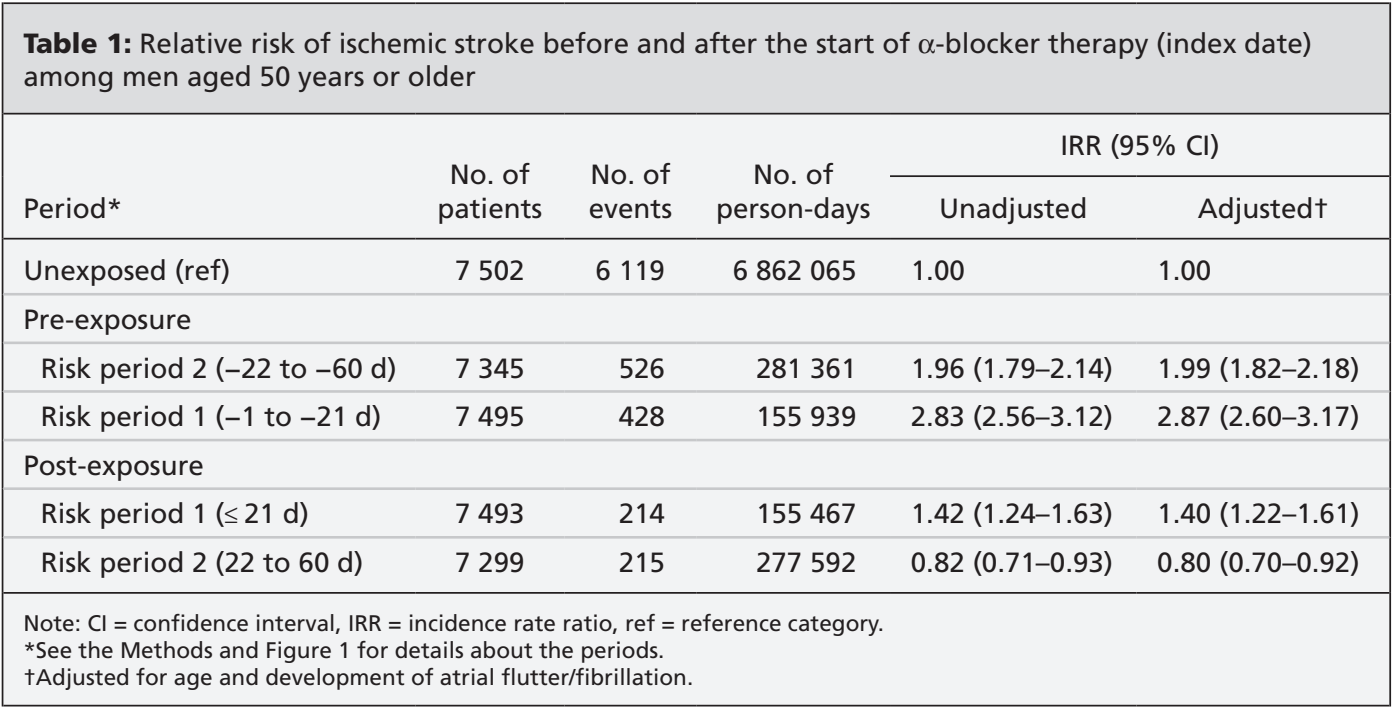


(adjusted IRR 1.07, 95\% CI 0.88-1.29), whereas the incidence in post-exposure risk period 2 was significantly lower than in the unexposed period (adjusted IRR 0.67, 95\% CI 0.56-0.81). In contrast, among patients without concomitant prescriptions for antihypertensive agents, the incidence of ischemic stroke was significantly higher in post-exposure risk period 1 than in the unexposed period (adjusted IRR 2.11, 95\% CI 1.73 2.57), whereas the incidence in post-exposure risk period 2 was similar to that in the unexposed period (adjusted IRR 1.06, 95\% CI 0.86-1.31). Irrespective of whether patients had concomitant prescriptions for antihypertensive agents, the incidence of ischemic stroke was significantly higher in both pre-exposure risk periods than in the unexposed period (Table 2).
Regardless of the type of $\alpha$-blocker administered, the adjusted IRRs of ischemic stroke were significantly elevated in both pre-exposure risk periods compared with the unexposed period (Table 3). In post-exposure risk period 1, the adjusted IRR remained significantly elevated for all of the $\alpha$-blockers studied except alfuzosin. In post-exposure risk period 2 , none of the $\alpha$-blockers was associated with an increased risk of ischemic stroke, and alruzosin and the standard formula of doxazosin were associated with a decreased incidence of ischemic stroke (Table 3).

In the first sensitivity analysis, the ischemic strokes that occurred on the same day as the index date of $\alpha$-blocker therapy $(n=1793$; $23.9 \%$ of the study population) were included in pre-exposure risk period 1 . In the second sensi-

\begin{tabular}{|c|c|c|c|c|}
\hline \multirow[b]{2}{*}{ Period* } & \multicolumn{2}{|c|}{$\begin{array}{c}\text { Concomitant use } \\
\text { of antihypertensive agents }\end{array}$} & \multicolumn{2}{|c|}{$\begin{array}{c}\text { No concomitant use } \\
\text { of antihypertensive agents }\end{array}$} \\
\hline & $\begin{array}{c}\text { Unadjusted IRR } \\
(95 \% \mathrm{Cl})\end{array}$ & $\begin{array}{c}\text { Adjusted IRR } † \\
(95 \% \mathrm{Cl})\end{array}$ & $\begin{array}{c}\text { Unadjusted IRR } \\
(95 \% \mathrm{CI})\end{array}$ & $\begin{array}{l}\text { Adjusted IRR }+ \\
(95 \% \mathrm{CI})\end{array}$ \\
\hline Unexposed (ref) & 1.00 & 1.00 & 1.00 & 1.00 \\
\hline \multicolumn{5}{|l|}{ Pre-exposure } \\
\hline Risk period 2 ( -22 to $-60 \mathrm{~d}$ ) & $2.01(1.80-2.24)$ & $2.05(1.84-2.28)$ & $1.85(1.57-2.17)$ & $1.89(1.61-2.22)$ \\
\hline Risk period 1 ( -1 to $-21 \mathrm{~d}$ ) & $2.91(2.58-3.28)$ & $2.94(2.61-3.32)$ & $2.66(2.22-3.18)$ & $2.71(2.27-3.24)$ \\
\hline \multicolumn{5}{|l|}{ Post-exposure } \\
\hline Risk period $1(\leq 21 \mathrm{~d})$ & $1.09(0.90-1.32)$ & $1.07(0.88-1.29)$ & $2.10(1.72-2.56)$ & $2.11(1.73-2.57)$ \\
\hline Risk period 2 ( 22 to $60 \mathrm{~d}$ ) & $0.69(0.58-0.83)$ & $0.67(0.56-0.81)$ & $1.07(0.87-1.31)$ & $1.06(0.86-1.31)$ \\
\hline
\end{tabular}

Table 3: Relative risk of ischemic stroke, by $\alpha$-blocker

\begin{tabular}{|c|c|c|c|c|c|c|c|c|c|c|}
\hline \multirow[b]{2}{*}{ Period* } & \multicolumn{2}{|c|}{ Terazosin } & \multicolumn{2}{|c|}{ Doxazosin } & \multicolumn{2}{|c|}{ Doxazosin-XL } & \multicolumn{2}{|c|}{ Tamsulosin } & \multicolumn{2}{|c|}{ Alfuzosin } \\
\hline & $\begin{array}{l}\text { Unadjusted } \\
\text { IRR }(95 \% \mathrm{CI})\end{array}$ & $\begin{array}{c}\text { Adjusted } \\
\text { IRRT }(95 \% \mathrm{Cl})\end{array}$ & $\begin{array}{l}\text { Unadjusted } \\
\text { IRR (95\% Cl) }\end{array}$ & $\begin{array}{c}\text { Adjusted } \\
\text { IRRT }(95 \% \mathrm{Cl})\end{array}$ & $\begin{array}{l}\text { Unadjusted } \\
\text { IRR }(95 \% \mathrm{CI})\end{array}$ & $\begin{array}{c}\text { Adjusted } \\
\text { IRRt }(95 \% \mathrm{Cl})\end{array}$ & $\begin{array}{l}\text { Unadjusted } \\
\text { IRR (95\% CI) }\end{array}$ & $\begin{array}{c}\text { Adjusted } \\
\text { IRRT }(95 \% \mathrm{Cl})\end{array}$ & $\begin{array}{l}\text { Unadjusted } \\
\text { IRR }(95 \% \text { CI) }\end{array}$ & $\begin{array}{c}\text { Adjusted } \\
\text { IRRT }(95 \% \mathrm{CI})\end{array}$ \\
\hline Unexposed (ref) & 1.00 & 1.00 & 1.00 & 1.00 & 1.00 & 1.00 & 1.00 & 1.00 & 1.00 & 1.00 \\
\hline \multicolumn{11}{|l|}{ Pre-exposure } \\
\hline $\begin{array}{l}\text { Risk period } 2 \\
(-22 \text { to }-60 \mathrm{~d})\end{array}$ & $\begin{array}{c}1.77 \\
(1.45-2.15)\end{array}$ & $\begin{array}{c}1.80 \\
(1.48-2.20)\end{array}$ & $\begin{array}{c}1.92 \\
(1.57-2.35)\end{array}$ & $\begin{array}{c}1.95 \\
(1.59-2.38)\end{array}$ & $\begin{array}{c}2.56 \\
(1.98-3.31)\end{array}$ & $\begin{array}{c}2.59 \\
(2.00-3.35)\end{array}$ & $\begin{array}{c}2.00 \\
(1.74-2.30)\end{array}$ & $\begin{array}{c}2.04 \\
(1.77-2.34)\end{array}$ & $\begin{array}{c}1.55 \\
(1.05-2.29)\end{array}$ & $\begin{array}{c}1.58 \\
(1.07-2.34)\end{array}$ \\
\hline $\begin{array}{l}\text { Risk period } 1 \\
(-1 \text { to }-21 \text { d) }\end{array}$ & $\begin{array}{c}2.80 \\
(2.27-3.45)\end{array}$ & $\begin{array}{c}2.85 \\
(2.31-3.52)\end{array}$ & $\begin{array}{c}2.55 \\
(2.03-3.20)\end{array}$ & $\begin{array}{c}2.57 \\
(2.04-3.23)\end{array}$ & $\begin{array}{c}3.36 \\
(2.49-4.52)\end{array}$ & $\begin{array}{c}3.39 \\
(2.52-4.56)\end{array}$ & $\begin{array}{c}2.85 \\
(2.44-3.32)\end{array}$ & $\begin{array}{c}2.88 \\
(2.47-3.36)\end{array}$ & $\begin{array}{c}2.89 \\
(1.97-4.25)\end{array}$ & $\begin{array}{c}2.94 \\
(2.00-4.32)\end{array}$ \\
\hline \multicolumn{11}{|l|}{ Post-exposure } \\
\hline $\begin{array}{l}\text { Risk period } 1 \\
(\leq 21 \text { d) }\end{array}$ & $\begin{array}{c}1.37 \\
(1.02-1.83)\end{array}$ & $\begin{array}{c}1.37 \\
(1.02-1.83)\end{array}$ & $\begin{array}{c}1.57 \\
(1.17-2.09)\end{array}$ & $\begin{array}{c}1.53 \\
(1.15-2.05)\end{array}$ & $\begin{array}{c}1.61 \\
(1.06-2.45)\end{array}$ & $\begin{array}{c}1.59 \\
(1.05-2.42)\end{array}$ & $\begin{array}{c}1.33 \\
(1.06-1.66)\end{array}$ & $\begin{array}{c}1.29 \\
(1.04-1.62)\end{array}$ & $\begin{array}{c}1.45 \\
(0.85-2.48)\end{array}$ & $\begin{array}{c}1.43 \\
(0.84-2.44)\end{array}$ \\
\hline $\begin{array}{l}\text { Risk period } 2 \\
\text { (22 to } 60 \mathrm{~d})\end{array}$ & $\begin{array}{c}0.79 \\
(0.59-1.06)\end{array}$ & $\begin{array}{c}0.79 \\
(0.59-1.05)\end{array}$ & $\begin{array}{c}0.65 \\
(0.46-0.91)\end{array}$ & $\begin{array}{c}0.64 \\
(0.46-0.89)\end{array}$ & $\begin{array}{c}1.12 \\
(0.76-1.63)\end{array}$ & $\begin{array}{c}1.10 \\
(0.75-1.62)\end{array}$ & $\begin{array}{c}0.90 \\
(0.74-1.11)\end{array}$ & $\begin{array}{c}0.88 \\
(0.72-1.08)\end{array}$ & $\begin{array}{c}0.41 \\
(0.19-0.86)\end{array}$ & $\begin{array}{c}0.40 \\
(0.19-0.85)\end{array}$ \\
\hline
\end{tabular}

Note: $\mathrm{Cl}=$ confidence interval, $\mathrm{IRR}=$ incidence rate ratio, ref = reference category, $\mathrm{XL}=$ modified-release formula. 
tivity analysis, we excluded the patients whose $\alpha$-blocker therapy was started during a hospital stay ( $n=3135 ; 41.8 \%$ of study population). Owing to the reduction in event numbers in the unexposed period compared with the design of the primary analysis, all of the IRRs of ischemic stroke in the 4 risk periods compared with the unexposed period were increased in both sensitivity analyses compared with the primary analysis (Tables S3-S6 in Appendix 1).

When we repeated the same design as the primary analysis among patients newly prescribed diuretics, we observed results similar to those of the primary analysis (Table S7 in Appendix 1). Among patients newly prescribed calcium-channel blockers, the risk of ischemic stroke was increased in post-exposure risk period 1 regardless of whether the patients had concomitant prescriptions for antihypertensive agents (Table S8 in Appendix 1).

\section{Interpretation}

In our study of claims data for more than 7500 older men in Taiwan prescribed an $\alpha$-blocker, we observed an increased risk of ischemic stroke in the early initiation period (within $21 \mathrm{~d}$ after the start of treatment) and a reduced risk in the late initiation period (22-60 d after the start of treatment). In our stratified analysis, only patients without concomitant exposure to other antihypertensive agents had an increased risk of ischemic stroke during the early initiation period of $\alpha$-blocker therapy. In contrast, patients already using antihypertensive agents had no increased risk of ischemic stroke in the early postexposure period and even had a decreased risk of stroke after treatment for more than 3 weeks. The increased risk of ischemic stroke during the early initiation period of $\alpha$-blocker therapy was observed for nearly all of the $\alpha$-blockers studied.

Alpha-blockers are notorious for their firstdose effect of acute hypotension during the early initiation period. ${ }^{5,7,8}$ Although several studies have shown that $\alpha$-blocker therapy could improve cerebral blood flow after treatment for 4-8 weeks, ${ }^{20,21}$ acute hypotension associated with antihypertensive therapy could precipitate cerebral hypoperfusion and ischemic stroke. ${ }^{2-4,11}$ One possible explanation of our findings was that patients without underlying hypertension were vulnerable to the first-dose effect of $\alpha$-blockers and contributed to the observed increase in risk of ischemic stroke in the early initiation period of $\alpha$-blockers in the whole study population. In contrast, patients with underlying hypertension appeared to be tolerant of the firstdose effect of $\alpha$-blockers, benefited from the positive influence of $\alpha$-blockers on cerebral blood flow and contributed to the observed decrease in risk of ischemic stroke in the late initiation period in the whole study population.

Significantly high IRRs in the 2 pre-exposure risk periods reflected the prescription pattern of $\alpha$-blocker therapy frequently being started after an ischemic stroke (Table S9 in Appendix 1). We could attribute this prescription pattern to the frequent development of high blood pressure during the episode of an acute ischemic stroke. ${ }^{22}$ However, this explanation could not be applied to tamsulosin and alfuzosin, the $2 \alpha$-blockers without an indication for use in patients with hypertension. Therefore, development of urinary symptoms after an ischemic stroke ${ }^{23}$ could be a better explanation of this prescription pattern, since most $\alpha$-blockers are currently prescribed for benign prostatic hyperplasia rather than hypertension., ${ }^{5,6}$

It has been reported that patients with stenosis of the intracranial carotid artery are more prone to ischemic stroke provoked by cerebral hypoperfusion than patients with stenosis of the extracranial carotid artery. ${ }^{24}$ Chinese people are at increased risk of intracranial carotid artery stenosis, and white people are at increased risk of extracranial carotid artery stenosis. ${ }^{25-27}$ Our findings were derived from claims data for mainly ethnic Chinese men in Taiwan. Further research is needed to determine whether the same findings would be observed in other ethnic populations. In addition, studies investigating the value of transcranial Doppler examination in the diagnosis of intracranial carotid artery stenosis ${ }^{28,29}$ and guidance of $\alpha$-blocker therapy are warranted.

In the ALLHAT study, patients in the doxazosin group had a slightly increased rate of stroke compared with those in the chlorthalidone group (relative risk $1.19,95 \%$ CI $1.01-1.40 ; p=0.04) .{ }^{6}$ In our study, the IRR of ischemic stroke during the early initiation period among patients without underlying hypertension was higher among those prescribed $\alpha$-blockers than among those prescribed diuretics (adjusted IRR 2.11 v. 1.80); therefore, our findings are in line with those from the ALLHAT study despite indirect comparison. Among patients prescribed calcium-channel blockers, the IRR of ischemic stroke was much higher in the early initiation period than in the unexposed period regardless of concomitant use of other antihypertensive agents. More pharmarcoepidemiologic studies are needed before the group effect of all hypertensive agents on acute cerebral hypoperfusion and acute ischemic stroke can be established.

\section{Limitations}

We did not include 5- $\alpha$-reductase inhibitors, an alternative treatment for benign prostatic hyperplasia, in our analysis owing to an inadequate 
sample size. Another limitation was our inability to determine from the claims data whether prescribed medications were taken by the patients.

\section{Conclusion}

The association between $\alpha$-blocker therapy and ischemic stroke was driven mostly by the prescription of $\alpha$-blockers following a previous ischemic stroke. However, our results presented evidence that $\alpha$-blockers were associated with an increase in the risk of ischemic stroke during the early initiation period, especially among patients who were not taking other antihypertensive agents. We recommend caution when prescribing $\alpha$-blockers to patients who are not taking other antihypertensive medications.

\section{References}

1. Adams HP Jr, Bendixen BH, Kappelle LJ, et al. Classification of subtype of acute ischemic stroke. Definitions for use in a multicenter clinical trial. TOAST. Trial of Org 10172 in Acute Stroke Treatment. Stroke 1993;24:35-41.

2. Klijn CJ, Kappelle LJ. Haemodynamic stroke: clinical features, prognosis, and management. Lancet Neurol 2010;9:1008-17.

3. Fischberg GM, Lozano E, Rajamani K, et al. Stroke precipitated by moderate blood pressure reduction. J Emerg Med 2000; 19:339-46.

4. Hankey GJ, Gubbay SS. Focal cerebral ischaemia and infarction due to antihypertensive therapy. Med J Aust 1987;146:412-4

5. Lepor H, Kazzazi A, Djavan B. Alpha-blockers for benign prostatic hyperplasia: The new era. Curr Opin Urol 2012;22:7-15.

6. ALLHAT Officers and Coordinators for the ALLHAT Collaborative Research Group. Major cardiovascular events in hypertensive patients randomized to doxazosin vs chlorthalidone: the Antihypertensive and Lipid-Lowering Treatment to Prevent Heart Attack Trial (ALLHAT). JAMA 2000;283:1967-75.

7. de Mey C. Cardiovascular effects of alpha-blockers used for the treatment of symptomatic BPH: impact on safety and wellbeing. Eur Urol 1998;34:18-28.

8. Lin MS, Hsieh WJ. Prazosin-induced first-dose phenomenon possibly associated with hemorrhagic stroke: a report of three cases. Drug Intell Clin Pharm 1987;21:723-6.

9. Chrischilles E, Rubenstein L, Chao J, et al. Initiation of nonselective alpha1-antagonist therapy and occurrence of hypotensionrelated adverse events among men with benign prostatic hyperplasia: a retrospective cohort study. Clin Ther 2001;23:727-43.

10. Lai CL, Kuo RN, Chen HM, et al. Risk of hip/femur fractures during the initiation period of $\alpha$-adrenoceptor blocker therapy among elderly males: a self-controlled case series study. $\mathrm{Br} J$ Clin Pharmacol 2015;80:1208-18.

11. Mansoor GA, Tendler BE. Stroke associated with alpha blocker therapy for benign prostatic hypertrophy. J Natl Med Assoc 2002; 94:1-4.

12. Lai CL, Wu CF, Kuo RN, et al. Clinical outcomes in low risk coronary artery disease patients treated with different limus-based drug-eluting stents - a nationwide retrospective cohort study using insurance claims database. PLoS ONE 2015;10:e0122860.

13. Whitaker HJ, Farrington CP, Spiessens B, et al. Tutorial in biostatistics: the self-controlled case series method. Stat Med 2006; 25:1768-97.

14. Whitaker HJ, Hocine MN, Farrington CP. The methodology of self-controlled case series studies. Stat Methods Med Res 2009; 18:7-26.

15. Best JH, Hoogwerf BJ, Herman WH, et al. Risk of cardiovascular disease events in patients with type 2 diabetes prescribed the glucagon-like peptide 1 (GLP-1) receptor agonist exenatide twice daily or other glucose-lowering therapies: a retrospective analysis of the lifelink database. Diabetes Care 2011;34:90-5.

16. Cheng CL, Kao YH, Lin SJ, et al. Validation of the National Health Insurance Research Database with ischemic stroke cases in Taiwan. Pharmacoepidemiol Drug Saf 2011;20:236-42.
17. Gribbin J, Hubbard R, Gladman J, et al. Risk of falls associated with antihypertensive medication: self-controlled case series. Pharmacoepidemiol Drug Saf 2011;20:879-84.

18. Pratt N, Roughead EE, Ramsay E, et al. Risk of hospitalization for hip fracture and pneumonia associated with antipsychotic prescribing in the elderly: a self-controlled case-series analysis in an Australian health care claims database. Drug Saf 2011;34:567-75.

19. Quan H, Sundararajan V, Halfon P, et al. Coding algorithms for defining comorbidities in ICD-9-CM and ICD-10 administrative data. Med Care 2005;43:1130-9.

20. Mochizuki Y, Oishi M, Takasu T. Effects of bunazosin hydrochloride sustained-release formulation on cerebral circulation. Int Angiol 2000;19:35-8.

21. Usuda K, Katayama Y. The effect of doxazosin mesilate on cerebral blood flow in patients with hypertension and chronic cerebral infarction. J Nippon Med Sch 2009;76:148-53.

22. Jauch EC, Saver JL, Adams HP Jr, et al. Guidelines for the early management of patients with acute ischemic stroke: a guideline for healthcare professionals from the American Heart Association/American Stroke Association. Stroke 2013;44:870-947.

23. Pilcher M, MacArthur J. Patient experiences of bladder problems following stroke. Nurs Stand 2012;26:39-46.

24. Chen H, Hong H, Liu D, et al. Lesion patterns and mechanism of cerebral infarction caused by severe atherosclerotic intracranial internal carotid artery stenosis. J Neurol Sci 2011;307:79-85.

25. Liu HM, Tu YK, Yip PK, et al. Evaluation of intracranial and extracranial carotid steno-occlusive diseases in Taiwan Chinese patients with MR angiography: preliminary experience. Stroke 1996;27:650-3.

26. Leung SY, Ng TH, Yuen ST, et al. Pattern of cerebral atherosclerosis in Hong Kong Chinese. Severity in intracranial and extracranial vessels. Stroke 1993;24:779-86.

27. Feldmann E, Daneault N, Kwan E, et al. Chinese-white differences in the distribution of occlusive cerebrovascular disease. Neurology 1990;40:1541-5.

28. Kidwell CS, el-Saden S, Livshits Z, et al. Transcranial Doppler pulsatility indices as a measure of diffuse small-vessel disease. J Neuroimaging 2001;11:229-35.

29. Ghorbani A, Ahmadi MJ, Shemshaki H. The value of transcranial Doppler derived pulsatility index for diagnosing cerebral smallvessel disease. Adv Biomed Res 2015;4:54.

Affiliations: Department of Internal Medicine and Center for Critical Care Medicine (C.-L. Lai), National Taiwan University Hospital Hsin-Chu Branch, Hsin-Chu, Taiwan; Department of Internal Medicine (C.-L. Lai), National Taiwan University College of Medicine, Taipei, Taiwan; Institute of Epidemiology and Preventive Medicine (C.-L. Lai, M.-S. Lai) and Institute of Health Policy and Management (Kuo), College of Public Health, National Taiwan University, Taipei, Taiwan; Center for Comparative Effectiveness Research (Kuo, H.-M. Chen, M.-S. Lai), National Center of Excellence for Clinical Trial and Research, National Taiwan University Hospital, Taipei, Taiwan; Departments of Internal Medicine (M.-F. Chen) and Medical Research (Chan), National Taiwan University Hospital, Taipei, Taiwan; Graduate Institute of Oncology (Chan), National Taiwan University College of Medicine, Taipei, Taiwan

Contributors: All of the authors contributed to the study conception and design and the data interpretation. Raymond Kuo and Ho-Min Chen conducted the statistical analysis. Chao-Lun Lai and K. Arnold Chan drafted the manuscript. All of the authors revised the manuscript for important intellectual content, approved the final version to be published and agreed to act as guarantors of the work.

Funding: This work was supported by funding (grants no. DOH101-TD-B-111-001 and DOH102-TD-B-111-001) from the Science and Technology Unit, Ministry of Health and Welfare, Executive Yuan, Taiwan.

Acknowledgement: All of the data used in this study were released and approved by the Collaboration Center of Health Information Application, Ministry of Health and Welfare, Executive Yuan, Taiwan. 